\title{
Visual performance of the intraindividual
}

\section{implantation of a trifocal intraocular lens in the bag and a +4.0 $\mathrm{D}$ bifocal intraocular lens in the sulcus with optic capture created by femtosecond laser-assisted cataract surgery}

This article was published in the following Dove Press journal: International Medical Case Reports Journal

\author{
Takeshi Teshigawara ${ }^{1-3}$ \\ Akira Meguro ${ }^{3}$ \\ Kazuro Yabuki ${ }^{4}$ \\ Seiichiro Hata ${ }^{5}$ \\ Nobuhisa Mizuki ${ }^{3}$ \\ 'Yokosuka Chuoh Eye Clinic, Yokosuka, \\ Kanagawa, Japan; ${ }^{2}$ Tsurumi Chuoh Eye \\ Clinic, Yokohama, Kanagawa, Japan; \\ ${ }^{3}$ Department of Ophthalmology, \\ Yokohama City University School \\ of Medicine, Yokohama, Kanagawa, \\ Japan; ${ }^{4}$ Department of Ophthalmology, \\ Saiseikai Yokohamashi Nanbu \\ Hospital, Yokohama, Kanagawa, Japan; \\ ${ }^{5}$ Yokohama Sky Eye Clinic, Yokohama \\ Kanagawa, Japan
}

\begin{abstract}
At present, only one design is available for trifocal intraocular lens (IOL); unfortunately, this particular design is not suitable for implantation in the sulcus with optic capture when posterior capsule rupture (PCR) occurs. Although three-piece bifocal IOLs can be implanted in the sulcus, this form of IOL can be vulnerable to tilt and decentration, thus causing aberration and photopic phenomena, such as halos and glares. However, visual axis centered optic capture using femtosecond laser-assisted cataract surgery (FLACS) is able to manage such complex operations.

In the present study, we implanted a three-piece +4.0 D bifocal IOL into the sulcus of a patient who experienced PCR using optic capture and FLACS following the straightforward implantation of a one-piece trifocal IOL in the other eye. Defocus curves showed that the weakness of the trifocal IOL (nearest distances) was compensated for by the strength of the +4.0 D bifocal IOL, whereas the weakness of the $+4.0 \mathrm{D}$ bifocal IOL (middle distance) was compensated for by the strength of the trifocal IOL. Therefore, this combination provided the patient with a wider range of depth of focus. The contrast sensitivity in both eyes was within the normal range. Photopic phenomena were comparable with the bilateral implantation of the trifocal IOL. Anterior segment optical coherence tomography showed that tilt and decentration in the trifocal IOL implanted in the bag was significantly higher than the $+4.0 \mathrm{D}$ bifocal IOL implanted in the visual axis centered optic capture.

This case showed that the intraindividual implantation of a single-piece trifocal IOL in the bag and a three-piece +4.0 D bifocal IOL in the sulcus, using a combination of optic capture and FLACS, is promising particularly in cases of PCR and can provide a wider range of vision without losing visual quality.

Keywords: multifocal intraocular lens, mixing and matching, optic capture, femtosecond laserassisted cataract surgery, posterior capsule rupture
\end{abstract}

\section{Introduction}

Various types of multifocal intraocular lens (IOL) have recently become available. It is important to be conversant with the pros and cons of IOLs. Single-piece trifocal IOLs have become popular since they provide three good focal points: distance, intermediate $(1 \mathrm{~m})$, and near vision $(40 \mathrm{~cm})$. In contrast, three-piece $+4.0 \mathrm{D}$ bifocal IOLs provide two focal points (distance and very near vision $[30 \mathrm{~cm}]$ ) and can be
Correspondence: Takeshi Teshigawara Yokosuka Chuoh Eye Clinic, 238-0008, 2-6 Odaki-cho, Yokosuka, Kanagawa, Japan

Tel +8 I 46827400 I

Fax $+8|468274| 4 \mid$

Email teshitake@gmail.com 
implanted in the sulcus using optic capture. ${ }^{1-3}$ However, each type of IOL has specific weaknesses. One of the weak points of trifocal IOLs is that only one design is currently available, which is not suitable for implantation in the sulcus when an undesirable event has occurred during operation. Another weak point is that the quality of very near vision for trifocal IOLs is slightly lower than +4.0 D bifocal IOLs. ${ }^{4}$ On the other hand, the typical weaknesses of +4.0 D bifocal IOLs include lower visual performance at intermediate distance, and photic phenomena, such as halos and glare. ${ }^{5}$ Under normal circumstances, implantation of a trifocal IOL in the bag in both eyes would be the most favorable option. Nevertheless, in certain circumstances, such as posterior capsule rupture (PCR), a second option needs to be prepared which meets patient expectation. In such cases, three-piece $+4.0 \mathrm{D}$ bifocal IOLs are available to implant into the sulcus; however, the performance of this type of IOL is still vulnerable to tilt and decentration and can induce even worse photic phenomena. Femtosecond lasers can create reproducible circularity and diameter during continuous curvilinear capsulorhexis (CCC). Consequently, implantation of three-piece $+4.0 \mathrm{D}$ bifocal IOLs using optic capture could represent an ideal option with which to manage such complex operations. This case report analyzed the visual performance outcome in a patient who agreed to have a three-piece $+4.0 \mathrm{D}$ bifocal IOL implanted in the sulcus using optic capture following a straightforward cataract operation with a single-piece trifocal in the bag.

\section{Case report}

A 68-year-old man arrived at our clinic with visually significant cataracts bilaterally. He had not received eye surgery previously. The preoperative corrected distance visual acuity was $20 / 30$ in the right eye and 20/20 in the left eye, with a refraction of 6.0 to $1.5 \times 140$ and -6.5 to $1.25 \times 150$, respectively.

Femtosecond laser-assisted cataract surgery (FLACS) with implantation of a one-piece trifocal diffractive IOL with double C-loop haptics (Pod F FINE VISION, PhysIOL, Liège, Belgium) (Figure 1) was scheduled for both eyes. In the right eye, a trifocal diffractive IOL was implanted following a straightforward FLACS procedure. The same procedure was planned for the left eye 1 week later. As planned, FLACS was performed using the femtosecond laser (LenSx, Alcon Laboratories, Fort Worth, TX, USA); $5.0 \mathrm{~mm}$ capsulotomy centered on White-to-White was created with $8.0 \mathrm{~mJ}$ of energy (spot and layer separations: $9 \mu \mathrm{m}$ each) (Figure 2A).

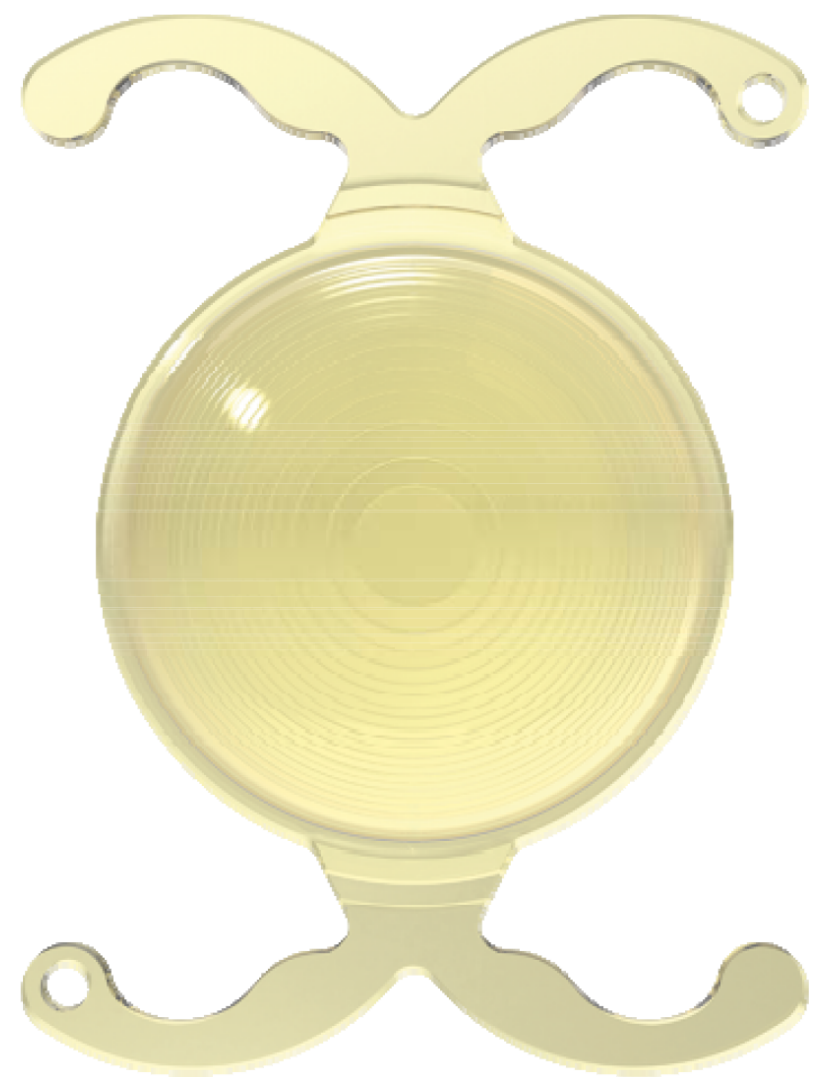

Figure I Photographic image of the single-piece trifocal IOL (Pod F FINE VISION). Abbreviation: IOL, intraocular lens.

Nuclear fragmentation was performed using the chop and cylinder technique with $8.0 \mathrm{~mJ}$ of energy (spot and layer separations: $9 \mu \mathrm{m}$ each) (Figure 2A). An optical coherence tomography (OCT) line scan indicated that there was no abnormality in the posterior capsule (Figure 2B). While the cortex was aspirated, the posterior capsule foramen developed at the 9 o'clock position (Figure 3). Anterior vitrectomy was performed to clear the vitreous from the anterior chamber and capsular bag. Since the foramen was located relatively peripherally, it was too large to implant a one-piece trifocal lens as planned. Consequently, a three-piece $+4.0 \mathrm{D}$ bifocal diffractive IOL with single C-loop haptics (Tecnis multifocal ZMA00, Abbott Medical Optics Inc., Santa Ana, CA, USA) (Figure 4) was placed in the sulcus using optic capture (Figure 5).

One month postoperatively, refraction was $+0.25-$ $0.25 \times 100$ in the right eye with the trifocal IOL and $0.00-0.25 \times 180$ in the left eye with the $+4.0 \mathrm{D}$ bifocal IOL, respectively. Uncorrected distance visual acuity was 30/20 in both eyes. Uncorrected intermediate visual acuity, uncorrected near visual acuity at $40 \mathrm{~cm}$, and the uncorrected near visual acuity at $30 \mathrm{~cm}$ were 20/20, 20/30, and 20/40, 


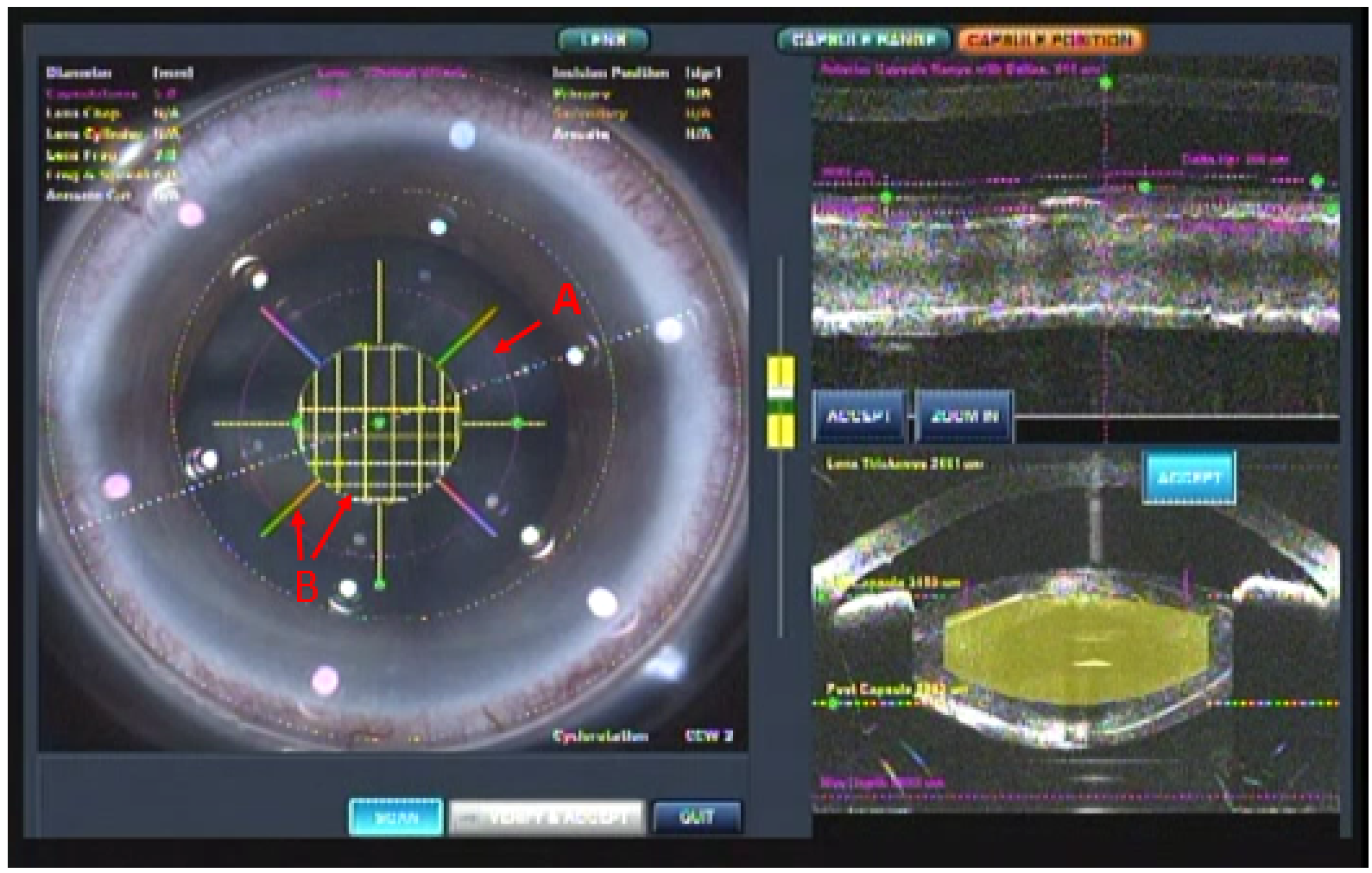

Figure 2 Intraoperative image taken during femtosecond laser surgery.

Notes: (A) $5.0 \mathrm{~mm}$ diameter CCC centered on White-to-White (pink circle). (B) Nuclear fragmentation using the cylinder and chop mode (yellow circle and eight pattern). Abbreviation: CCC, curvilinear capsulorhexis.

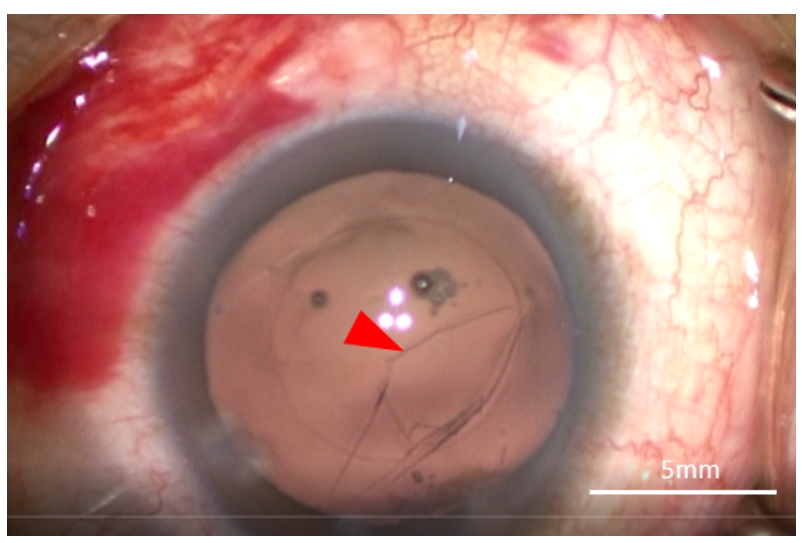

Figure 3 Image of the development of the posterior capsule foramen at the 9 o'clock position (red arrow).

respectively, in the right eye with the trifocal IOL. These were $20 / 20,20 / 40$, and 20/25, respectively, in the left eye with the $+4.0 \mathrm{D}$ bifocal IOL. The defocus curve in each eye was similar to the one in the trifocal IOL and the bifocal IOL, which were provided by the manufacturers (Figure 6A). A binocular defocus curve showed that the strength of each IOL compensated for the weaknesses in each type of IOL (Figure 6B). As a matter of fact, the patient's satisfaction with each distance was high, and he noticed that the intermediate distance was covered by the right eye, whereas, the near distance of $30 \mathrm{~cm}$ was covered by the left eye. A contrast sensitivity test (Contrast Glaretester, Takagi, Japan) indicated that the contrast sensitivity in both eyes was within the normal range (Figure 7). Anterior segment OCT (CASIA2, Tomey, Nagoya, Japan) showed that the degree of both tilt (degree) and decentration $(\mathrm{mm})$ in the single-piece trifocal IOL implanted in the bag $(9.9$ and $0.49 \mathrm{~mm})$ was higher than the three-piece bifocal IOL implanted in the sulcus using optic capture (2.3 and $0.10 \mathrm{~mm}$ ) (Figure 8).

The patient provided informed written consent for the publication of his case details, and the accompanying images.

\section{Discussion}

This intraindividual research study analyzed the implantation of a one-piece trifocal IOL in the bag, and a three-piece bifocal IOL in the sulcus, using optic capture created by a femtosecond laser. Our findings indicated good visual outcomes and high patient satisfaction.

The trifocal IOL provides better intermediate visual acuity than the bifocal IOL in addition to good far and near vision. ${ }^{6}$ Initially, we planned to implant the trifocal IOL into 


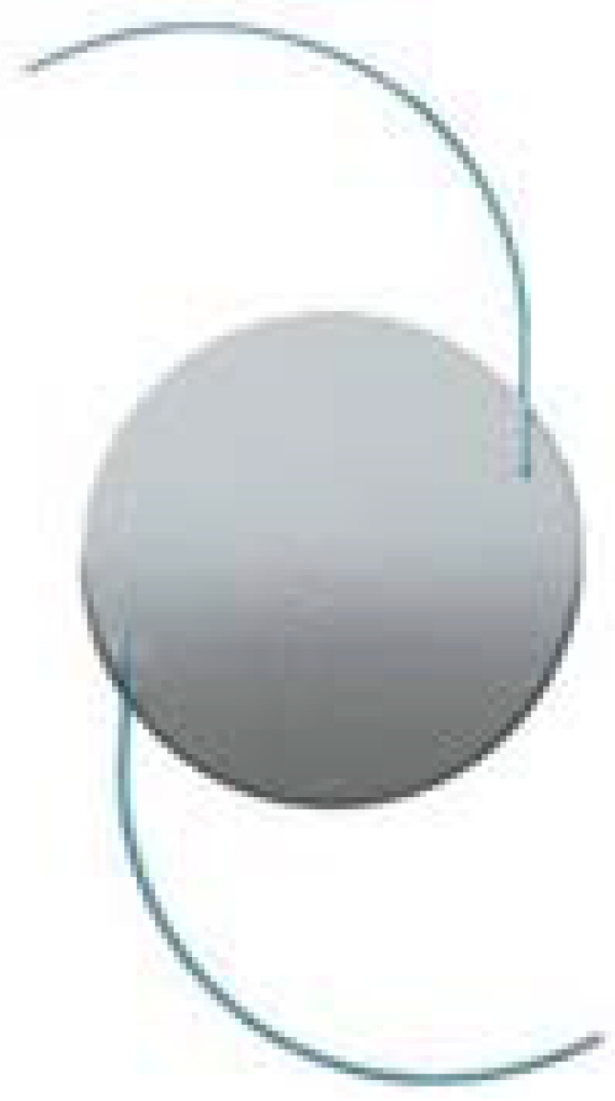

Figure 4 Photographic image of the three-piece +4.0 D bifocal IOL (ZMA00). Abbreviation: IOL, intraocular lens.

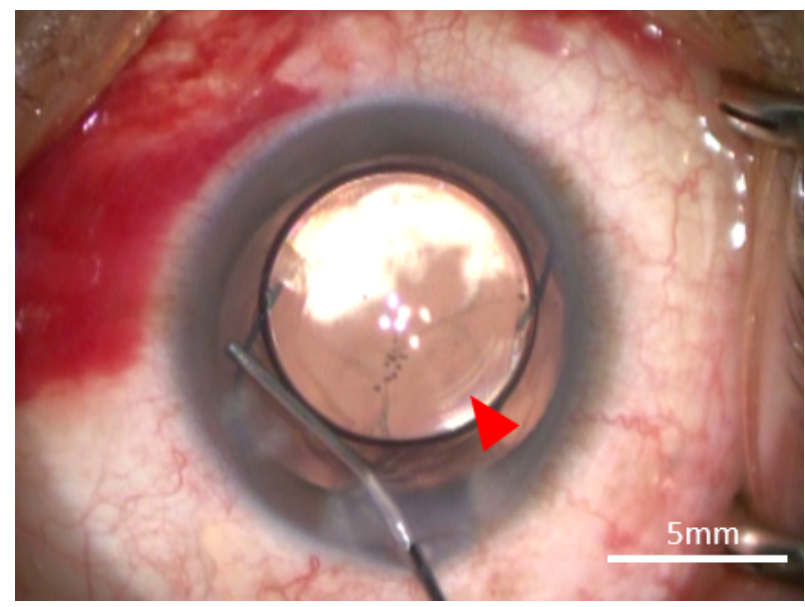

Figure 5 Image of optic capture involving the three-piece $+4.0 \mathrm{D}$ bifocal IOL. Note: The CCC is indicated by the red arrow.

Abbreviations: CCC, curvilinear capsulorhexis; IOL, intraocular lens.

each eye. A trifocal IOL was placed into the bag in the right eye as planned. Unexpectedly, however, PCR occurred during the operation in the left eye; therefore, we had to discuss the choice of which IOL to implant based on their individual pros and cons. A one-piece trifocal diffractive IOL with double C-loop haptics limits implantation into an intact bag or a bag with a posterior CCC. ${ }^{7}$ However, in our case, the PCR occupied a large area superiorly; it was therefore risky to create a posterior CCC to implant the trifocal IOL in the bag. Therefore, one option for implantation was a three-piece monofocal IOL or a bifocal IOL in the sulcus. ${ }^{7}$ Technically speaking, a monofocal IOL is more suitable to place into the sulcus because the quality of vision of a bifocal IOL is more vulnerable to tilt and decentration. ${ }^{8-10}$ Optic capture is a valid option with which to deal with such circumstances. ${ }^{10,11}$ In the case of unforeseen events, such as our case, the implantation of a three-piece bifocal IOL into the sulcus using optic capture should be considered. Fortunately, since this cataract operation was performed by FLACS, the CCC was a suitable size and was round-shaped on the capsular bag. For this specific situation, the best choice for patient satisfaction was the implantation of a three-piece +4.0 D bifocal diffractive IOL with single C-loop haptics into the sulcus using optic capture.

While it is quite challenging for a manual procedure to achieve a completely round-shaped, well-centered and similarly-sized (ideal diameter for optic capture $=5 \mathrm{~mm}$ ) capsulotomy, ${ }^{12}$ FLACS enables us to overcome this challenge. ${ }^{12}$ In contrast to the manual procedure, FLACS can create a CCC with reproducible circularity and diameter in a desirable position within the anterior capsule. ${ }^{13}$ As was the situation with our present case, FLACS can widen the range of IOL choice, even under complicated situations with a capsular bag.

Regarding the quality of vision, in our case, both the right eye with the trifocal IOL, and the left eye with the +4.0 D bifocal IOL, showed an almost identical defocus curve to the ones provided by the manufacturers. In a previous study, Mojzis et a ${ }^{14}$ demonstrated that there was no significant difference in distant vision when compared between trifocal and $+4.0 \mathrm{D}$ bifocal IOLs. Our present case also showed comparable findings in terms of distant vision. Like most previous studies, ${ }^{3,15,16}$ our present case demonstrated that the right eye with the trifocal IOL showed better intermediate vision $(1 \mathrm{~m})$ than the left eye with the +4.0 D bifocal IOL; the very near vision $(30 \mathrm{~cm})$ of the left eye was better than the right eye. The near vision $(40 \mathrm{~cm})$ of the right eye was comparable to that of the left eye. This result indicated that in the defocus curve, the strengths of each IOL could compensate for the weaknesses of each other, which was evident in the binocular defocus curve. Cochener ${ }^{17}$ also presented comparable binocular visual outcome following the implantation of a trifocal IOL in one eye and a bifocal IOL in the other eye.

Previous research has indicated that a multifocal IOL can cause a significant reduction in contrast sensitivity compared to a monofocal IOL. ${ }^{18-21}$ In our case, the right eye with the 

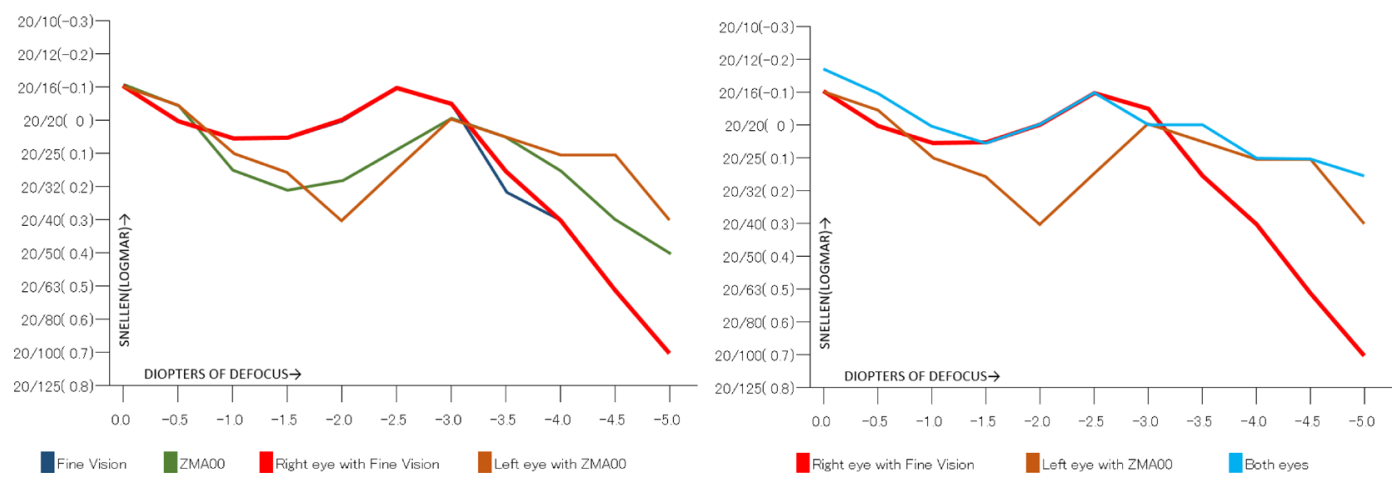

Figure 6 (A) Defocus curve of the Fine Vision trifocal IOL (navy) ZMA00 bifocal IOL (green); right eye with the trifocal IOL (red) and left eye with the bifocal IOL (brown). The defocus curve for the Fine Vision trifocal IOL (navy) and ZMA00 bifocal IOL (green) was provided by the manufacturers. (B) Defocus curve for the right eye with the Fine Vision trifocal IOL (red); left eye with the ZMA00 bifocal IOL (brown) and both eyes (light blue).

Abbreviation: IOL, trifocal intraocular lens.

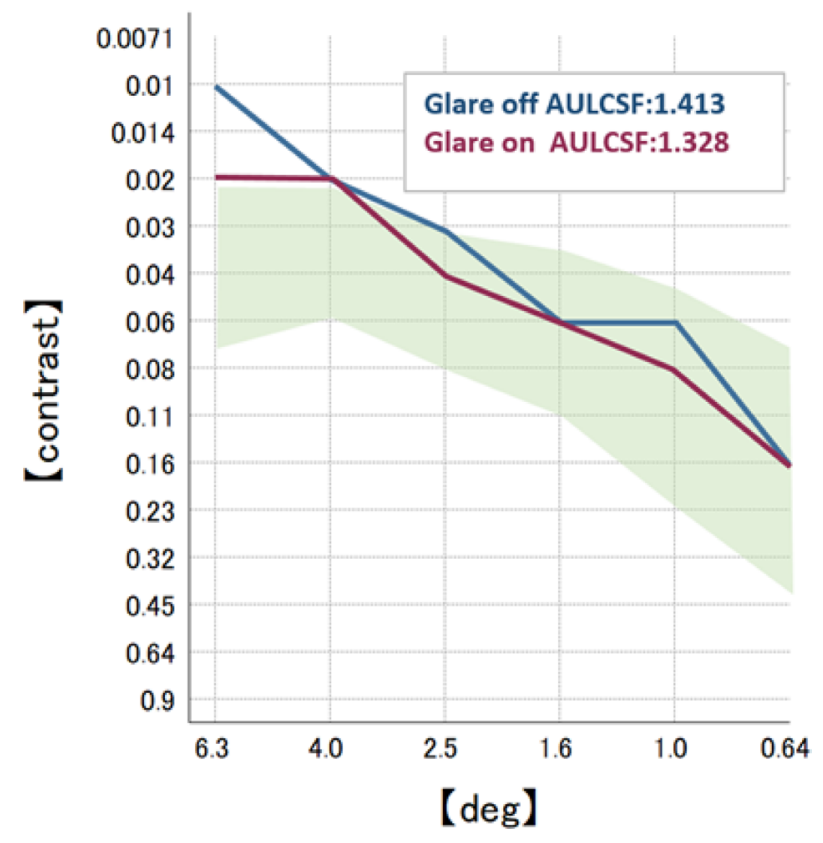

Figure 7 Postoperative contrast sensitivity in both eyes. Note: The shaded area indicates the normal range.

trifocal IOL showed a slightly higher area under the log contrast sensitivity function than the left eye in the $+4.0 \mathrm{D}$ bifocal IOL. This difference, however, was not statistically significant, and both results were above average. This indicates that contrast sensitivity of the implantation of the trifocal and +4.0 D bifocal IOL in each eye can be comparable in a case in which the trifocal IOL was implanted in both eyes.

Photic phenomena, such as halos and glare, are also a major concern after a multifocal IOL implantation. ${ }^{22,23}$ While Al-Khateeb et al ${ }^{24}$ found that these symptoms were more severe in a bifocal IOL than a trifocal IOL, Cochener ${ }^{17}$ showed different results in which symptoms were more troublesome in a trifocal IOL than a bifocal IOL. Our case showed that both halos and glares in the right eye with the trifocal IOL were slightly milder than in the left eye with the +4.0 D bifocal IOL. In both eyes, the halos and glares were comparable with bilateral implantation of the trifocal IOL. In the patient's questionnaire, there was no indication of issues relating to photic phenomena in everyday life, including night driving.

When it comes to the stabilization of refraction, optic capture can perform earlier anterior-posterior stabilization than in-the-bag IOL placement. Indeed, in-the-bag IOL placement can cause delayed myopic shift more frequently than optic capture ${ }^{25}$ Stitfter et a ${ }^{26}$ reported that in most cases, in-the-bag IOL placement can show forward movement in the first few weeks after operation, and forward or backward movement between 3 months and 1 year postoperation. This phenomena can cause continuous refractive change; such a tendency was also seen in our case. While refraction in the right eye with the single-piece trifocal IOL in the bag showed myopic shift for 6 months postoperation, the left eye, implanted with the three-piece bifocal IOL in the sulcus using optic capture, demonstrated no refractive shift after the operation. Consequently, the refraction with optic capture is most probably stabilized sooner than that with in-the-bag IOL placement, which can offer more accurate postoperative refraction. ${ }^{27}$ FLACS can consistently offer an accurate diameter of CCC which enables us to place IOLs in the sulcus using optic capture without overstretching the capsule and risking a tear in the capsule. Furthermore, some studies have reported that IOL implantation by optic capture in the center of the visual axis shows better visual outcome than others. ${ }^{28}$ While it is not a problem for experienced surgeons to create a CCC of the same size consistently, the FLACS method is superior to the 

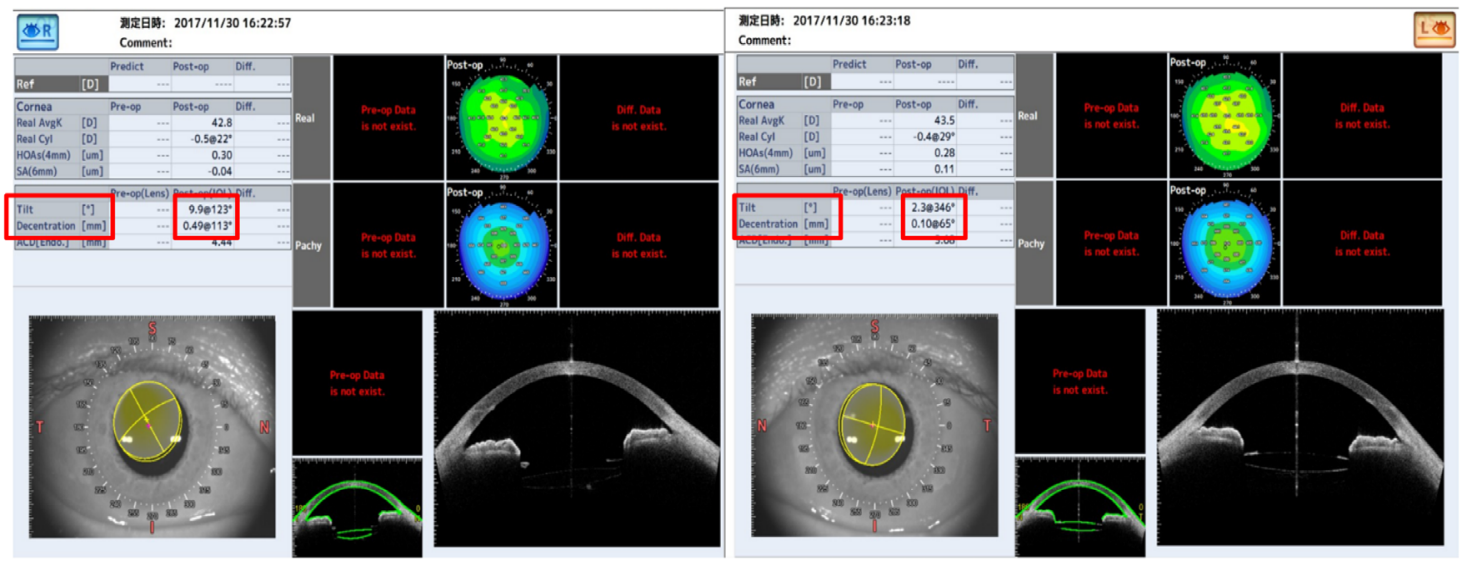

Figure 8 Image of the anterior segment OCT.

Notes: R: right eye with the single-piece trifocal IOL in the bag. L: left eye with the three-piece bifocal IOL in the sulcus of the optic capture. Degree of tilt and decentration is indicated by the red box.

Abbreviations: IOL, intraocular lens; OCT, optical coherence tomography.

manual procedure in that it can consistently produce a $\mathrm{CCC}$ on the visual axis. Given the fact that FLACS enables us to create optic capture at a desirable position, even in our case, optic capture should have been positioned in the center of the visual axis, rather than the center of the White-to-White. As a matter of fact, the recently released IOL, known as FEMINIS (Oculentis, Berlin, Germany), is specially designed to implant into the bag with optic capture in the center of the visual axis created by FLACS, and is expected to show better performance in terms of the quality of vision. ${ }^{29}$

In conclusion, our present case showed that intraindividual implantation of a trifocal IOL in the bag, and a +4.0 D bifocal IOL in the sulcus, using optic capture created by FLACS is a promising technique, since the strength of each IOL supplemented their own weaknesses, thus leading to a wider depth of focus. This is particularly useful for when unanticipated PCR occurs because it ensures that the outcome of the operation can still live up to patient expectation. Furthermore, FLACS can play an important role in performing this technique in a consistent and predictable manner.

\section{Acknowledgment}

All researchers involved in this case study have no affiliations with or involvement in any organization or entity with any financial interest or nonfinancial interest.

\section{Disclosure}

The authors report no conflicts of interest in this work.

\section{References}

1. Mojzis P, Kukuckova L, Majerova K, Liehneova K, Piñero DP. Comparative analysis of the visual performance after cataract surgery with implantation of a bifocal or trifocal diffractive IOL. J Refract Surg. 2014;30(10):666-672.

2. Kaymak H, Breyer D, Alió JL, Cochener B. Visual performance with bifocal and trifocal diffractive intraocular lenses: a prospective three-armed randomized multicenter clinical trial. J Refract Surg. 2017;33(10):655-662.

3. Gatinel D, Houbrechts Y. Comparison of bifocal and trifocal diffractive and refractive intraocular lenses using an optical bench. $J$ Cataract Refract Surg. 2013;39(7):1093-1099.

4. Maxwell A, Holland E, Cibik L, et al. Clinical and patient-reported outcomes of bilateral implantation of a +2.5 diopter multifocal intraocular lens. J Cataract Refract Surg. 2017;43(1):29-41.

5. Carson D, Lee S, Alexander E, Wei X, Lee S. Comparison of two laboratory-based systems for evaluation of halos in intraocular lenses. Clin Ophthalmol. 2018;12:385-393.

6. Kohnen T, Titke C, Böhm M, B€ohm M. Trifocal intraocular lens implantation to treat visual demands in various distances following lens removal. Am J Ophthalmol. 2016;161:71-77.

7. Al-Khateeb G, Shajari M, Kohnen T. Intraindividual comparative analysis of the visual performance after cataract surgery with implantation of a trifocal and a bifocal intraocular lens. J Cataract Refract Surg. 2017;43(5):695-698.

8. Eppig T, Scholz K, Löffler A, Meßner A, Langenbucher A. Effect of decentration and tilt on the image quality of aspheric intraocular lens designs in a model eye. J Cataract Refract Surg. 2009;35(6):1091-1100.

9. Sauer T, Mester U. Tilt and decentration of an intraocular lens implanted in the ciliary sulcus after capsular bag defect during cataract surgery. Graefes Arch Clin Exp Ophthalmol. 2013;251(1):89-93.

10. Yang HC, Chung SK, Baek NH, Decentration BNH. Decentration, tilt, and near vision of the Array multifocal intraocular lens. $J$ Cataract Refract Refractive Surgery. 2000;26(4):586-589.

11. Gimbel HV, Amritanand A. Reverse optic capture to stabilize a toric intraocular lens. Case Rep Ophthalmol. 2013;4(3):138-143.

12. Friedman NJ, Palanker DV, Schuele G, et al. Femtosecond laser capsulotomy. J Cataract Refract Surg. 2011;37(7):1189-1198.

13. Nagy Z, Takacs A, Filkorn T, Sarayba M. Initial clinical evaluation of an intraocular femtosecond laser in cataract surgery. $J$ Refract Surg. 2009;25(12):1053-1060. 
14. Mojzis P, Kukuckova L, Majerova K, Liehneova K, Piñero DP. Comparative analysis of the visual performance after cataract surgery with implantation of a bifocal or trifocal diffractive IOL. J Refract Surg. 2014;30(10):666-672.

15. Zhao G, Zhang J, Zhou Y, Hu L, Che C, Jiang N. Visual function after monocular implantation of apodized diffractive multifocal or single-piece monofocal intraocular lens. J Cataract Refract Surg. 2010;36(2):282-285.

16. Nuijts RMMA, Jonker SMR, Kaufer RA, et al. Bilateral implantation of +2.5 D multifocal intraocular lens and contralateral implantation of +2.5 D and +3.0 D multifocal intraocular lenses: Clinical outcomes. $J$ Cataract Refract Surg. 2016;42(2):194-202.

17. Cochener B. Prospective clinical comparison of patient outcomes following implantation of trifocal or bifocal intraocular lenses. J Refract Surg. 2016;32(3):146-151.

18. Pieh S, Weghaupt H, Skorpik C. Contrast sensitivity and glare disability with diffractive and refractive multifocal intraocular lenses. J Cataract Refract Surg. 1998;24(5):659-662.

19. Cillino S, Casuccio A, di Pace F, et al. One-year outcomes with new-generation multifocal intraocular lenses. Ophthalmology. 2008;115(9):1508-1516.

20. Kohnen T, Allen D, Boureau C, et al. European multicenter study of the AcrySof ReSTOR apodized diffractive intraocular lens. Ophthalmology. 2006;113(4):578-584.

21. Sheppard AL, Shah S, Bhatt U, Bhogal G, Wolffsohn JS. Visual outcomes and subjective experience after bilateral implantation of a new diffractive trifocal intraocular lens. J Cataract Refract Surg. 2013;39(3):343-349.
22. de Vries NE, Webers CAB, Touwslager WRH, et al. Dissatisfaction after implantation of multifocal intraocular lenses. J Cataract Refract Surg. 2011;37(5):859-865.

23. Sood P, Woodward MA. Patient acceptability of the Tecnis multifocal intraocular lens. Clin Ophthalmol. 2011;5:403-410.

24. Al-Khateeb G, Shajari M, Kohnen T. Intraindividual comparative analysis of the visual performance after cataract surgery with implantation of a trifocal and a bifocal intraocular lens. $J$ Cataract Refract Refractive Surgery. 2017;43(5):695-698.

25. Menapace R. Posterior capsulorhexis combined with optic buttonholing: an alternative to standard in-the-bag implantation of sharp-edged intraocular lenses? A critical analysis of 1000 consecutive cases. Graefes Arch Clin Exp Ophthalmol. 2008;246(6):787-801.

26. Stifter E, Menapace R, Luksch A, Neumayer T, Sacu S. Anterior chamber depth and change in axial intraocular lens position after cataract surgery with primary posterior capsulorhexis and posterior optic buttonholing. J Cataract Refract Surg. 2008;34(5):749-754.

27. Scott WJ, Owsiak RR. Femtosecond laser-assisted primary posterior capsulotomy for toric intraocular lens fixation and stabilization. J Cataract Refract Surg. 2015;41(8):1767-1771.

28. Versace P. Angle kappa as a determinant of success with multifocal IOLs. Paper presented at: The XXXIV Congress of the ESCRS; September 10-14; 2017; Copenhagen, Denmark.

29. Cummings A. Capsulorhexis reconsidered. Eurotimes Stories. Available from: http://www.eurotimes.org/capsulorhexis-reconsidered/. Accessed September 10, 2018
International Medical Case Reports Journal

\section{Publish your work in this journal}

The International Medical Case Reports Journal is an international, peer-reviewed open-access journal publishing original case reports from all medical specialties. Previously unpublished medical posters are also accepted relating to any area of clinical or preclinical science. Submissions should not normally exceed 2,000 words or

\section{Dovepress}

4 published pages including figures, diagrams and references. The manuscript management system is completely online and includes a very quick and fair peer-review system, which is all easy to use. Visit http://www.dovepress.com/testimonials.php to read real quotes from published authors. 